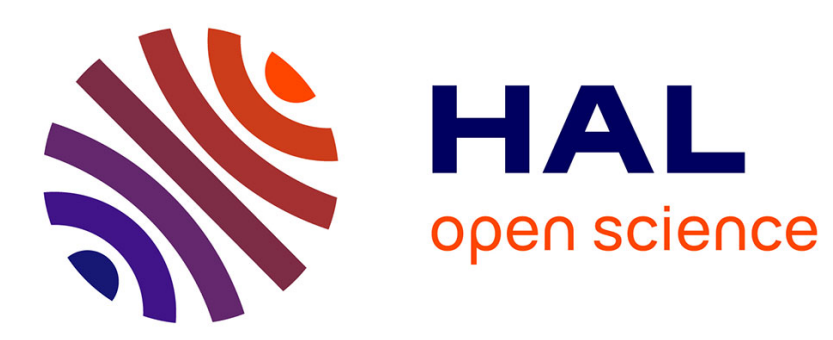

\title{
Les monnaies sociales en tant que dispositifs innovants: une évaluation
}

\author{
Jérôme Blanc, Marie Fare
}

\section{To cite this version:}

Jérôme Blanc, Marie Fare. Les monnaies sociales en tant que dispositifs innovants: une évaluation. Innovations - Revue d'économie et de management de l'innovation, 2012, 2012/2 (38), pp.67-84. 10.3917/inno.038.0067 . halshs-00704263

\section{HAL Id: halshs-00704263 \\ https://shs.hal.science/halshs-00704263}

Submitted on 19 Apr 2021

HAL is a multi-disciplinary open access archive for the deposit and dissemination of scientific research documents, whether they are published or not. The documents may come from teaching and research institutions in France or abroad, or from public or private research centers.
L'archive ouverte pluridisciplinaire HAL, est destinée au dépôt et à la diffusion de documents scientifiques de niveau recherche, publiés ou non, émanant des établissements d'enseignement et de recherche français ou étrangers, des laboratoires publics ou privés. 


\section{LES MONNAIES SOCIALES EN TANT QUE DISPOSITIFS INNOVANTS : UNE ÉVALUATION}

Jérôme Blanc et Marie Fare

De Boeck Supérieur | Innovations

$2012 / 2-n^{\circ} 38$
pages 67 à 84

ISSN 1267-4982

Article disponible en ligne à l'adresse:

http://www.cairn.info/revue-innovations-2012-2-page-67.htm

Pour citer cet article :

Blanc Jérôme et Fare Marie, « Les monnaies sociales en tant que dispositifs innovants : une évaluation », Innovations, 2012/2 n³8, p. 67-84. DOI : 10.3917/inno.038.0067

Distribution électronique Cairn.info pour De Boeck Supérieur.

(C) De Boeck Supérieur. Tous droits réservés pour tous pays.

La reproduction ou représentation de cet article, notamment par photocopie, n'est autorisée que dans les limites des conditions générales d'utilisation du site ou, le cas échéant, des conditions générales de la licence souscrite par votre établissement. Toute autre reproduction ou représentation, en tout ou partie, sous quelque forme et de quelque manière que ce soit, est interdite sauf accord préalable et écrit de l'éditeur, en dehors des cas prévus par la législation en vigueur en France. II est précisé que son stockage dans une base de données est également interdit. 


\title{
LES MONNAIES SOCIALES EN TANT QUE DISPOSITIFS INNOVANTS : UNE ÉVALUATION ${ }^{1}$
}

\author{
Jérôme BLANC \\ Triangle UMR 5206, Université Lumière Lyon 2 \\ Institut des Sciences de l'Homme (ISH) \\ jerome.blanc@univ-lyon2.fr \\ Marie FARE \\ Triangle UMR 5206, Université Lumière Lyon 2 \\ Institut des Sciences de l'Homme (ISH) \\ marie.fare@univ-lyon2.fr
}

Les monnaies sociales sont citées de manière croissante lorsqu'il s'agit d'illustrer les dynamiques innovantes en économie solidaire, aux côtés d'initiatives de commerce équitable, de circuits alimentaires courts, de finance solidaire ou de microcrédit. Elles sont sans doute parmi les moins connues et comprises de ces initiatives, sans doute parce que leur émergence a été moins rapide et spectaculaire que les autres, que leur signification et leurs objectifs apparaissent plus difficiles à identifier et que la mobilisation d'outils monétaires heurte d'emblée une représentation sociale forte, celle de la souveraineté.

Il s'agit de dispositifs d'échange locaux de biens, de services et de savoirs, organisés autour d'une monnaie spécifique permettant à la fois d'évaluer et de régler les échanges. On parle aussi de "monnaies complémentaires », de «monnaies locales », de «monnaies communautaires », parfois de «monnaies libres »; selon les langues, certains termes sont privilégiés ; de manière générale, ces choix terminologiques font sens dans la mesure où ils renvoient à des différences dans les objectifs et dans les moyens qui peuvent être considérables. Nous n'entrerons pas ici dans cette discussion et, par commodité,

1. Ce texte a bénéficié de nombreux commentaires, dans le cadre d'une journée d'études sur l'innovation sociale (Lyon, $1^{\mathrm{e}}$ mars 2010), des Xe Rencontres du Réseau Inter-universitaire de l'économie sociale et solidaire (RIUESS) (Luxembourg, 3-4 juin 2010) et du 13th World Congress of the Association for Social Economics (ASE) (Montréal, 28 juin-1 ${ }^{\mathrm{e}}$ juillet 2010). Nous remercions l'ensemble des personnes qui ont permis d'améliorer ce texte, tout en demeurant, bien entendu, seuls responsables des propos tenus et des erreurs possibles. 
nous considérerons ces dispositifs sous le terme générique de «monnaies sociales », conservant à l'esprit les difficultés que pose ce terme, au même titre que tout autre ${ }^{2}$. Ces dispositifs se sont développés dans plus de 50 pays depuis le début des années 1980 et il y en aurait aujourd'hui plus de 4000 dans le monde. Leur diversité reste méconnue ${ }^{3}$. Ils se déploient autour d'un nombre de modèles d'abord restreint (LETS, banques de temps dans les années 1980) qui s'est progressivement élargi (dans les années 1990, réseaux de Trueque sur le modèle argentin, monnaies Hour sur le modèle d'Ithaca ; dans les années 2000, monnaies de type Regio sur le modèle allemand, monnaies et banques communautaires sur le modèle de Fortaleza, monnaie à projets multiples comme la monnaie SOL en France, monnaies dites locales complémentaires en France, monnaies locales de «villes en transition » en Grande-Bretagne, systèmes de type RES, etc.).

Cette vague de monnaies est inédite à l'échelle mondiale depuis les débuts de l'industrialisation au tournant du 19 e siècle, et la progressive diversification des modèles existants depuis une trentaine d'années tout autant que la pérennité de cette vague posent la question du sens de ces dispositifs nouveaux. Bien qu'elles incluent parfois des dimensions technologiques évidentes (paiements par cartes à puce ou téléphones mobiles, plateformes internet, codes à bulle sur des billets...), c'est dans la construction de réponses locales à des besoins sociaux locaux, et donc dans des formes d'innovation sociale, que ces monnaies trouvent un socle commun.

L'innovation sociale n'est pas un concept récent mais il connaît un renouveau depuis les années 1970 (pour une revue de la littérature, voir Nussbaumer et Moulaert, 2007) malgré ou grâce à la multi-dimensionnalité de ses acceptions. L'innovation sociale se nourrit d'innovations de natures diverses: en matière technologique (au sens des techniques ou des outils employés pour une fin donnée), dans le domaine organisationnel (au sens de l'organisation de l'activité productive, en particulier l'organisation du travail), dans le rapport aux institutions publiques (au sens de la mise en contact, ou de la mobilisation, d'une combinaison nouvelle d'institutions existantes, ou de la promotion de la création d'institutions nouvelles) et dans la mobilisation de règles nouvelles (au sens institutionnaliste large de normes, valeurs et règles qui guident et parfois déterminent comportements

\footnotetext{
2. Pour une discussion des termes ainsi que des typologies en la matière, voir Blanc $(2006,2011)$.

3. Il est vrai cependant qu'aucune évaluation satisfaisante n'existe à l'heure actuelle, et l'on peut s'interroger sur la possibilité même d'une évaluation étant donné l'hétérogénéité de ces dispositifs. Le site http://www.complementarycurrency.org/ccDatabase/ fournit une vue d'ensemble, même partielle, de cette diversité.
} 
et pratiques). Mais l'innovation sociale ne saurait être une simple combinaison d'autres formes d'innovation au sens où elle produit des solutions inédites ayant une utilité sociale s'inscrivant dans un cadre normatif novateur. C'est ce que nous allons essayer de montrer au travers des monnaies sociales. Nous commençons par dresser un portrait rapide de la vague contemporaine de monnaies sociales, en montrant dans quelle mesure les modalités de sa diffusion incluent la possibilité d'innovations. Nous passerons ensuite en revue quatre dimensions qui paraissent caractériser l'innovation sociale et que l'on retrouve dans les monnaies sociales: l'importance des finalités et l'ancrage local, des logiques partenariales autour d'un projet fédérateur, une critique du modèle dominant et l'émergence de règles et de formes novatrices.

\section{UN DOUBLE PROCESSUS DE MULTIPLICATION ET DE DIFFÉRENCIATION PAR L'INNOVATION}

Après avoir identifié les modalités principales de multiplication et de différenciation des monnaies sociales et les possibilités induites d'innovation, nous passerons en revue quelques cas emblématiques de monnaies sociales dans lesquels apparaissent clairement des formes d'innovation.

\section{Modalités de la multiplication et de la différenciation}

La vague contemporaine de monnaies sociales commence avec la fondation, en 1983, du système LET'S de Comox Valley, sur l'île de Vancouver, au Canada, dans un contexte de chômage massif provoqué par la fermeture d'une industrie locale. Par la suite, l'appellation LETS est largement diffusée et est généralement comprise comme Local Exchange Trading System. L'histoire qui suit ce moment de fondation prend la forme d'un double mouvement de multiplication et de différenciation, dont la figure 1 rend compte sous forme chronologique. 
Figure 1 - Chronologie simplifiée des grands types de monnaies sociales

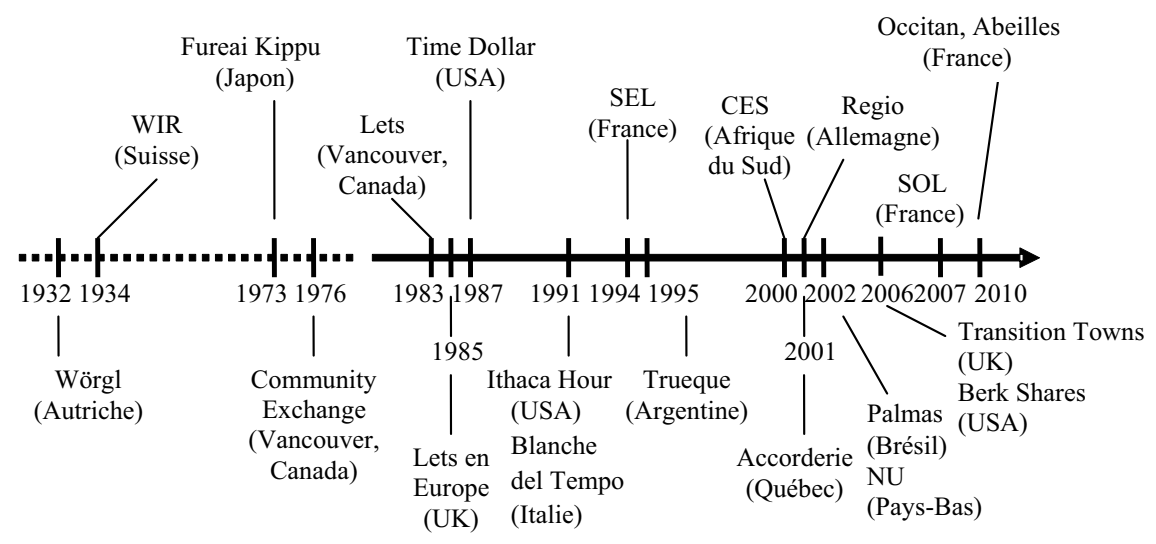

La typologie des modèles de développement établie par Pache et Chalencon (2007) à partir d'une étude sur les entreprises sociales permet de mieux comprendre ce double mouvement. Celui-ci combine les formes de "dissémination », « d'essaimage souple », « d'essaimage par franchise » et de « développement centralisé ».

(1) La multiplication des dispositifs d'un pays à l'autre a lieu essentiellement sous la forme de «dissémination », dans laquelle les créateurs de monnaies sociales mettent à disposition des informations sur leur dispositif, dont peuvent s'emparer ensuite toutes personnes intéressées pour lancer leur propre projet, qu'aucun lien formel ne reliera au(x) modèle(s) préalables. Les créateurs, cependant, peuvent jouer un rôle actif dans cette dissémination par des déplacements, interventions, etc. Ce mode de dissémination correspond bien au passage des LETS anglo-saxons aux SEL (systèmes d'échange local) français.

(2) Par un « essaimage souple », de nouveaux projets se développent de manière autonome mais à partir d'un cadre constituant un réseau avec par exemple la signature d'une charte commune. Notons que, dans le cas des monnaies sociales, le cadre peut être très souple, comme par exemple l'appartenance tacite à un réseau et la participation à des rencontres locales ou nationales où s'expriment certes les convergences, mais aussi potentiellement les divergences entre dispositifs. L'essaimage souple est le mode principal de diffusion à l'intérieur d'un pays, dans lequel les dispositifs locaux ont un grand intérêt à s'allier au sein de réseaux plus ou moins formels (SEL'idaire pour les SEL, le réseau des « monnaies locales complémentaires » pour les monnaies locales gravitant autour du modèle de l'Abeille, à Villeneuve-sur-Lot). 
(3) Certains rares dispositifs se développent par « essaimage en franchise », où le contrôle des dispositifs locaux par la structure fondatrice première est fort et passe par une contractualisation. Il s'agit là de répliquer un modèle tout en conservant le contrôle d'éléments fondamentaux du projet afin d'éviter toute dénaturation locale qui pourrait rejaillir sur les autres. Ce modèle peut avoir été mis en œuvre localement et fait ses preuves (cas de l'Accorderie de Québec), ou avoir d'abord été théorisé et mis en œuvre de manière centralisée (cas de la monnaie SOL depuis 2010).

(4) Le SOL a en effet d'abord été mis en œuvre par impulsion nationale et centralisée. Il renvoie donc davantage, jusqu'à 2010 tout du moins, à la quatrième modalité de diffusion : celle du «développement centralisé » où les structures locales n'ont pas d'autonomie juridique et très peu de capacité innovatrice propre - ce qui ne signifie pas que le SOL en tant que tel n'est pas novateur : il l'est assurément, mais d'abord dans sa conception centralisée.

L'innovation apparaît ainsi à deux niveaux. D'une part, elle peut intervenir comme différenciation majeure lors de la création d'un dispositif d'une nature nouvelle, soit dans le cadre d'un développement centralisé où l'adaptation aux conditions locales est résiduelle, soit dans le cadre d'une dissémination qui rend possible l'adaptation forte aux contraintes et besoins locaux. D'autre part, elle peut intervenir comme différenciation mineure lors de la multiplication des dispositifs par dissémination, essaimage souple ou en franchise.

Ces dynamiques expliquent que, en se diffusant, ces dispositifs se différencient : certes par des innovations qui visent une adaptation aux conditions locales, mais aussi par l'activation d'une culture de l'expérimentation et par la prise de conscience que la monnaie est un outil malléable que l'on peut adapter à des fins qu'il appartient aussi à la société civile de définir. Il s'agit maintenant de caractériser plus précisément les différenciations, mineures et majeures intervenues jusqu'ici. Le modèle fondateur des LETS apparaît à ce titre comme modèle de référence pour beaucoup des dispositifs ultérieurs.

\section{Différenciations à l'œuvre}

En 1983, le LET'S de Comox Valley qui est créé sur l'impulsion de Michael Linton, un Canadien d'origine écossaise, s'inspire de deux systèmes préexistants dont il se différencie par le recours à un logiciel informatique de gestion des échanges (par la tenue des comptes) et par l'établissement d'une monnaie interne à la fois à parité avec le dollar canadien et inconvertible, le Green dollar. Le LET'S est un système de crédit mutuel, où un échange se traduit par le débit d'un compte et le crédit d'un autre, la somme des soldes individuels étant toujours comptablement nul. Il n'est donc aucunement besoin d'émettre des moyens de paiement en amont des échanges, et ceux-ci 
se traduisent par de simples jeux d'écriture. Ce LETS ouvert aux entreprises entraîne la création d'une vingtaine de LETS en Amérique du nord, mais s'effondre au bout de deux ans et demi de fonctionnement, notamment du fait du blocage des échanges par deux causes : l'accumulation d'une dette excessive d'un adhérent, et l'accumulation de soldes positifs par les entreprises adhérentes, incapables de les utiliser. Ce premier échec conduira beaucoup des systèmes suivants à intégrer une limitation du solde maximal du compte de chacun, en déficit comme en excédent ${ }^{4}$.

Une seconde vague de LETS les conduit en Europe et en Océanie, sous forme de dissémination et d'essaimage souple (où Michael Linton et quelques autres militants jouent un rôle majeur de diffusion des informations et des idées) ${ }^{5}$, permettant des différenciations mineures en fonction des enjeux locaux, des difficultés légales etc. En France, les SEL, apparus en 1994, illustrent cette possibilité de différenciation mineure. Ils ont été peu à peu recentrés sur le temps comme mode normal d'évaluation des montants des échanges (ce qui ne fournit pas de règle simple pour les échanges de biens...), la convivialité comme objectif central et une exclusion d'échanges économiques professionnels ou semi-professionnels (Servet, 1999 ; Laacher, 2003).

Le principe de crédit mutuel des LETS se retrouve aussi dans les systèmes de banques de temps qui ont émergé à partir de 1987 aux États-Unis sous le nom de Time dollar. Ces «banques» sont cependant issues d'une différenciation majeure des LETS, car elles reposent sur des objectifs spécifiques. Elles visent notamment à stimuler une entraide sociale intergénérationnelle en rétribuant sous forme d'heures de services le temps passé par des personnes à aider des malades, personnes âgées ou autres personnes en demande d'aide. L'unité de compte interne est donc strictement l'heure de temps et les échanges portent uniquement sur des services. Obtenir des crédits de temps permet ainsi de bénéficier du temps des autres. Ces dispositifs sont souvent articulés à des collectivités publiques ou des financeurs extérieurs dès lors qu'ils sont considérés comme des plateformes d'entraide sociale. Selon Cahn, fondateur du modèle des Time dollars, elles sont suffisamment différentes des LETS pour qu'il n'y ait pas de concurrence possible (Cahn, 2001, 2004).

L'échec local d'un projet de LETS, ou ses difficultés, peut aussi conduire à imaginer des dispositifs nouveaux, comme dans le cas de l'Ithaca hour, créé en 1991 à Ithaca, petite ville de l'État de New York, et des systèmes de Trueque en Argentine à partir de 1995. Un élément clé à l'origine de ces deux

4. Dans de nombreux SEL français, le solde toléré est de 2000 unités locales en positif comme en négatif, ce qui limite du même coup le volume maximum d'un seul échange. Toutefois, pour permettre certains échanges, il est parfois décidé que le règlement peut être fractionné, cf. Servet (1999).

5. Voir notamment, à propos des débuts des LETS et sur la « doctrine » de Linton, Seron (1995). 
expériences est le refus du système purement scriptural des LETS impliquant une centralisation des informations auprès d'un service spécialisé dans la gestion des comptes ${ }^{6}$. L'usage de billets évite cette lourdeur. Quelques années plus tard, le système sud-africain CES (Community Exchange System, qui essaime de manière souple très au-delà de l'Afrique du Sud) innovera en combinant Internet et téléphonie mobile pour organiser de manière plus fluide, décentralisée et légère la gestion des comptes d'un LETS.

L'Ithaca hour est la conséquence directe de l'échec d'un LETS et d'une réflexion sur les causes de cet échec ${ }^{7}$. L'usage de billets partiellement convertibles et utilisables conjointement au dollar facilite les transactions de petits montants auprès de professionnels (sont particulièrement visés ici des fermiers de la région participant aux marchés et les commerces locaux) mais aussi démultiplie les possibilités d'extension du système dès lors qu'il n'est plus nécessaire d'être adhérent pour employer cette monnaie. Enfin, les billets donnent la possibilité d'un jeu graphique permettant de diffuser un message et des symboles.

Dans le modèle argentin du Trueque, le point de départ est aussi la création d'un LETS, puis son arrêt au profit d'un système à billets, cependant inconvertibles (DeMeulenaere, 2000). À la différence de l'Ithaca Hour, le Trueque se déploie dans un espace autonome, sous la forme de ferias (foires) régulières tenues par des bénévoles et dans des lieux a priori neutres du point de vue commercial. Pour y échanger, il faut adhérer, être formé au fonctionnement du nodo (le club local) et recevoir des billets permettant d'échanger en tant que "prosommateur ", c'est-à-dire à la fois producteur (obtenant la monnaie interne par la vente) et consommateur (dépensant ainsi la monnaie) (Luzzi, 2005). Il est remarquable que les dynamiques du Trueque ont rapidement été celles de l'essaimage en franchise, freinant largement l'innovation à l'intérieur même de chacun des réseaux franchisés.

Le Trueque argentin s'est disséminé au Brésil dès les années 1990, sous l'impulsion notamment de l'argentino-brésilienne Heloisa Primavera. Celle-ci a en outre été sollicitée durant la phase de maturation de l'idée de monnaie sociale dans une banlieue défavorisée de Fortaleza, le Conjunto Palmeira, pilotée par le Banco Palmas, banque communautaire endogène développant un système de microcrédit depuis quelques années dans le cadre de l'association de développement des habitants, l'ASMOCONP (Melo, 2009). La confrontation de ce dispositif fondé sur le concept de prosommateur, dans

6. Par exemple la gestion des comptes des membres du LETS australien de Katoomba, qui ont été jusqu'à 2000 dans les années 1990, a occupé jusqu'à une vingtaine de personnes.

7. Voir Douthwaite (1996) et Jacob, Brinkerhoff, Jovic et Wheatley (2004). On peut aussi consulter le site de Paul Glover traitant de l'expérience d'Ithaca (www.lightlink.com/ithacahours). 
lequel les besoins et les offres doivent donc être diversifiés, aux contraintes locales où la demande était d'abord celle de moyens de survie alimentaire et l'offre très réduite, a conduit à imaginer autre chose. Avec l'appui momentané de l'ONG néerlandaise Strohalm, un projet de monnaie locale a été mis en œuvre (Ferreira, Moers, 2006) puis pérennisé. La banque émet donc une monnaie spécifique, le Palma, notamment par conversion de reales et dans le cadre de crédits à la consommation, tout en réalisant aussi des crédits à la production en reales dont le remboursement doit être réalisé pour partie en palmas. Le Palma est au pair avec le real et utilisable localement uniquement et vise à créer une dynamique productive endogène et un circuit local de production, distribution de revenus et consommation apte à réduire la pauvreté dans le quartier. La réussite exemplaire du Banco Palmas a conduit à la création d'un Institut Palmas, dont le rôle, en partenariat avec le secrétariat national à l'économie solidaire, est de soutenir et stimuler la réplication du modèle sur le mode d'un essaimage en franchise. En 2011, on compte plus de 62 banques communautaires établies au Brésil dans ce cadre.

La monnaie SOL, mise en œuvre à partir de 2007 en France, représente un cas très particulier de monnaie sociale ${ }^{8}$. Elle articule un système de fidélisation de la clientèle appliqué à des entreprises, associations, commerces agréés pour leur orientation sociale et environnementale ( $\mathrm{SOL}$ coopération), un système de monnaie affectée à des usages définis par les politiques sociales des collectivités locales ( $\mathrm{SOL}$ affecté) et un système de valorisation de l'engagement associatif bénévole inspiré des SEL et des banques de temps (SOL engagement). Le projet prend sa source dans un travail de réflexion engagé par et autour de Patrick Viveret en 1998 qui a conduit à étudier les «monnaies plurielles». Il s'est concrétisé autour d'un programme Equal (financé par le Fonds Social Européen) fournissant un élan et des ressources financières suffisantes pour organiser de façon centralisée un projet technique et coûteux (Fare, 2007). C'est pourquoi le mode de développement de la monnaie SOL a d'abord été centralisé, les projets la mettant en œuvre sur les territoires n'ayant pas d'existence juridique propre ni d'autonomie technique - le projet Equal exigeant, en revanche, que des financements locaux soient levés pour $30 \%$ du budget total. Dans un second temps, à partir de 2009-10, le projet a été amendé et les territoires ont acquis davantage d'autonomie, juridique (par la création d'associations locales) et technologique (par la possibilité de déroger à la carte à puce) ; le mode de développement a donc été partiellement décentralisé au profit d'un essaimage en franchise.

8. Plus que pour les autres des cas présentés ci-dessus, la dénomination «monnaie sociale » est critiquable concernant le SOL, du fait de la multiplicité de ses logiques et inspirations ; mais pour les raisons mentionnées en introduction nous maintenons ici ce terme. 
Nous venons de montrer de quelle façon les différents dispositifs de monnaies sociales sont capables à la fois de s'inspirer des dispositifs antérieurs mais aussi d'innover. Il s'agit maintenant d'approfondir l'analyse en discutant de la nature de ces innovations. Nous montrerons en quoi les monnaies sociales constituent des formes d'innovations sociales.

\section{LES MONNAIES SOCIALES COMME INNOVATIONS SOCIALES : UNE VUE TRANSVERSALE}

\section{L'importance des finalités et de l'ancrage local}

L'innovation sociale se distingue d'autres types d'innovations par son objet, sa finalité qui lui confère son caractère « social ». Chambon, David et Deverey (1982) définissent les innovations sociales comme des «pratiques visant plus ou moins directement à permettre à un individu - ou à un groupe d'individus - de prendre en charge un besoin social - ou un ensemble de besoins - n'ayant pas trouvé de réponses satisfaisantes par ailleurs ». L'innovation sociale vise à répondre à des besoins ou des aspirations non satisfaits par le marché ou l'État. Ce sont donc des solutions inédites ayant une utilité sociale pour répondre à des attentes sociales émergentes (Bouchard, 2006a). L'anglais met en avant l'idée de grassroots innovations (par exemple, Seyfang et Smith, 2007) : il s'agit d'innovations menées par les acteurs eux-mêmes, et non pas dans un processus descendant top / down. À cette échelle, les acteurs du terrain ont la capacité de saisir des signaux faibles, inaudibles par les entreprises à but lucratif et par les pouvoirs publics.

Il en résulte une mise en avant de la capacité de proposition et d'invention issue de la société civile et sa capacité à s'approprier des problèmes et des solutions. Provenant de communautés ou d'associations de la société civile, les démarches entreprises se situent originellement hors des collectivités publiques et des démarches commerciales lucratives. L'innovation sociale se situe ainsi dans les interstices laissés par le marché et l'État qu'elle cherche à combler. Cela ne signifie cependant pas que la construction des réponses aux besoins et aspirations locales soit totalement et par principe hors des activités marchandes et des pouvoirs publics...

Ici besoins et aspirations sont fortement corrélés. Par exemple, les banques de temps cherchent à répondre au délitement du lien social et au désengagement de l'État, ce qui correspond à une demande sociale forte. Mais, en même temps, elles valorisent les compétences de chacun, ce qui peut s'assimiler à de nouvelles aspirations en termes de reconnaissance des activités domestiques. 
Il y a donc potentiellement articulation d'une logique palliative (améliorer l'existant) et d'une logique contestataire (contester les règles et valeurs en vigueur). Il peut donc apparaître un objectif en corollaire, plus ambitieux, ciblant une remise en cause du modèle de développement pouvant aboutir à une transformation ou un renouvellement du système économique et social, comme nous le soulignerons plus loin. Le LETS de Comox Valley s'inscrit dans cette perspective : il naît dans un contexte de crise aiguë, à la suite de la fermeture de l'industrie minière, qui suscite de nombreuses tentatives de « retour à la terre » ainsi que de multiples expériences coopératives et communautaires. Ainsi les LETS apparaissent comme un moyen de faire émerger des échanges compatibles avec des objectifs de reviviscence des territoires, déclinés notamment au travers de la sécurité collective basée sur la solidarité et les échanges de proximité, de la réduction de la dépendance par rapport aux monopoles de production, de distribution et de transport, de l'enracinement individuel dans un milieu naturel régénéré et entretenu, de modes de vie basés sur la complémentarité bénéfique à tous et non sur la compétitivité destructrice et de nouveaux espaces de créativité.

Outre la finalité, l'identification des besoins ou des aspirations par les acteurs, l'innovation sociale est fortement ancrée dans un territoire de proximité. Chambon, David et Deverey (1982) considèrent ainsi que si l'innovation sociale résulte de la recherche de solutions à des besoins ou des aspirations non satisfaits, celle-ci se construit et émerge localement. RichezBattesti (2008) montre qu'innovation sociale et territoire ont un lien très fort qui peut être appuyé sur différentes formes de proximité. Bouba-Olga et Grossetti (2008) distinguent une proximité spatiale d'une proximité socioéconomique. Alors que la proximité spatiale renvoie à «des distances physiques, des temps de transport, des coûts de transport, des coûts de communication ", la proximité socio-économique renvoie à "l'enchevêtrement des formes socioéconomiques qui structurent les échanges et les activités ", ou à ce qui rapproche les individus: des ressources (matérielles et cognitives) et des capacités de coordination (via des réseaux sociaux et des ressources de médiation telles que des journaux ou des sites internet). En ce sens, le territoire est un espace où d'autres formes de proximités sont susceptibles de se déployer et d'être employées comme ressources à des fins de projets collectifs. La monnaie étant par définition un fait social reliant les personnes, la mise en œuvre d'un projet de monnaie sociale puis sa réussite nécessite d'actionner les leviers de ces formes de proximité.

Tous les cas de monnaies sociales présentés dans ce texte sont mis en œuvre par des associations locales ad hoc en réponse à des aspirations et des besoins qui ne sont pas satisfaits par la production marchande et par la production publique. Même la monnaie SOL, qui a une ampleur nationale et a 
passé par un partenariat à un niveau supranational (Union européenne) et national (grandes organisations de l'économie sociale) n'échappe pas à cette règle lorsqu'il s'agit de la mettre en place concrètement : ce sont des acteurs locaux, qui depuis 2009-2010 tendent à se structurer en associations locales et montent les partenariats locaux permettant d'organiser le système et de l'adapter aux conditions locales.

Cette construction d'une réponse par la société civile est la condition qui permet tout à la fois la perception de ces besoins et la construction d'une réponse adaptée. La construction de la réponse elle-même apparaît dans une certaine mesure comme la réponse recherchée, en tant qu'elle suppose la construction d'un espace de délibération collective autour de règles économiques, alors que les règles économiques ordinaires sont précisément considérées comme inadaptées et insupportables. On peut souligner dans un certain nombre de cas le rôle central des espaces délibératifs mais aussi des espaces de rencontre entre usagers ou adhérents qui ne sont pas formellement délibératifs mais fournissent les conditions de bonne mise en œuvre de la participation de chacun : dans les LETS, les SEL, le Trueque et dans une certaine mesure le SOL. Il ne faut cependant pas nier la possibilité et dans un certain nombre de cas l'existence d'une coupure entre les usagers et la direction des dispositifs une fois établis, comme le souligne par exemple avec regret Melo (2009) pour le dispositif de Banco Palmas à Fortaleza, ou comme cela a pu être exprimé lors d'une assemblée générale de l'association nationale SOL en 2009, entre adhérents situés sur les territoires d'expérimentation et dirigeants de l'association.

L'Accorderie fournit un exemple type de construction d'une réponse endogène à un besoin social local. À la fin des années 1990, deux organismes québécois, la Caisse d'économie solidaire Desjardins et la Fondation St-Roch de Québec, ont engagé une réflexion sur la lutte contre la pauvreté et l'exclusion. Partant des constats, pour la Caisse d'économie solidaire Desjardins, de problèmes d'accès aux services bancaires, et pour la Fondation St-Roch de Québec, de problèmes de sécurité alimentaire, ils assemblent leurs compétences, au vu de la complémentarité de ces deux problématiques et d'une même approche, en créant un organisme répondant à ces deux objectifs, l'Accorderie. Un dispositif hybride et polyvalent voit le jour à l'automne 2001, sous la forme d'un système d'échange de services basé sur le temps, un dispositif de crédit solidaire et un groupement d'achat. C'est le système d'échange de services qui est à la base du fonctionnement de l'Accorderie et qui constitue son cœur d'activité. Cette réponse endogène à des besoins locaux se traduit en outre par la mise en œuvre d'une gouvernance participative démocratique originale (Fare, 2009-2010). 


\section{Les logiques partenariales autour d'un projet fédérateur}

En tant que dynamique sociale locale, l'innovation sociale s'inscrit dans une dynamique collective nécessitant une gouvernance partenariale (RichezBattesti, 2008). C'est ainsi qu'une autre caractéristique de l'innovation sociale consiste à mobiliser des ressources et des acteurs divers autour d'un projet fédérateur. La mise en œuvre de cette réponse nécessite la coordination et la coopération des acteurs.

Avant la phase collective de mise en œuvre d'une innovation sociale telle que les monnaies sociales, le processus de mobilisation des acteurs résulte généralement d'un fondateur militant. La notion de militance est ici importante. Créer un dispositif de monnaie sociale est en effet un acte de militance, au minimum dans son sens de militance associative. Alter (2002) considère d'ailleurs que ce sont des acteurs déviants qui impulsent les innovations. Le fondateur de dispositif est quelqu'un qui en général dispose d'un réseau relationnel dense, tissé au fil d'années de fréquentation du milieu associatif, de réflexions et de débats (Blanc, 2002). Le fondateur a des liens tels qu'il peut d'une part s'appuyer sur quelques personnes pour organiser le dispositif et d'autre part solliciter et motiver plusieurs personnes pour intégrer le dispositif dès sa création. L'extension du dispositif tient en outre, au départ, à la capacité de cet ensemble de personnes à diffuser l'information dans leurs propres réseaux relationnels. Le militant fondateur idéal-typique est ainsi une personne se trouvant à la croisée de plusieurs réseaux. Enfin, les militants fondateurs sont souvent des personnages, au sens de caractères ou figures emblématiques. Ils ont une capacité à impulser une dynamique forte aux dispositifs et parfois à incarner très personnellement les dispositifs. Plusieurs de ces figures ont été citées plus haut : Linton (LETS), Cahn (Time banking), Glover (Ithaca hour), Primavera (Trueque), Melo (Banco Palmas), Viveret (SOL). Le militant fondateur a un rôle fondamental par sa capacité à impulser la coopération en mobilisant les acteurs sur un projet risqué (car innovant).

Les acteurs de cette coopération sont divers. Le SOL, par exemple, ne peut se développer qu'à partir de la synergie entre des entreprises représentatives des valeurs de l'économie sociale et solidaire, des associations développant des actions d'entraide et créant du lien social, des collectivités territoriales mettant en œuvre des politiques contribuant à un développement qualitatif, humain et soutenable et des « consom'acteurs » donnant du sens à leurs achats et l'ensemble des personnes s'engageant dans des activités à caractère solidaire. La monnaie cristallise cet assemblage complexe.

D'autres dispositifs développent des partenariats avec des banques locales comme l'Ithaca Hour aux États-Unis ou le Chiemgauer allemand, ou avec une banque nationale comme le réseau des banques communautaires 
brésiliennes. Enfin, dans de rares cas, l'État central a été appelé à légiférer (Argentine sans succès; Venezuela, Brésil, Équateur) voire à soutenir le déploiement de ces dispositifs (Venezuela, Brésil, Équateur). Le développement des monnaies sociales peut donc conduire à des transformations réglementaires affirmant leur place.

Ainsi, il est difficile de considérer que les monnaies sociales sont par définition hors de l'univers marchand (voire lucratif) et hors de l'État : il s'agit bien plutôt d'agir dans leurs marges, et (de plus en plus) souvent de manière connectée. Ce qu'analyse Laville (dir. 2007, 2010) sous la dénomination d'économie solidaire est ici pertinent pour un certain nombre de dispositifs : l'hybridation des ressources et les logiques partenariales peuvent être au cœur des dispositifs de monnaies sociales.

\section{Une critique du " modèle dominant "}

Certaines innovations sociales se posent en opposition vis-à-vis du modèle dominant de la consommation et de la production de masse. Il peut s'agir de dépasser les frontières entre le développement économique et le développement social (Bouchard, 2006b), de lutter contre l'ordre établi, de remettre en cause les institutions, de prendre en compte l'environnement... L'innovation sociale peut ainsi être analysée comme une réaction face au modèle de développement et apparaître comme un témoin ou un révélateur de ces tensions. Elle s'inscrit dans le sillage des nouveaux mouvements sociaux nés dans les années 1970 et 1980 et qui cherchent à répondre à une nouvelle demande sociale, celle d'un autre ou d'un nouveau modèle sociétal. Au-delà des nouveaux mouvements sociaux, l'approche des «nouveaux mouvements sociaux économiques » (Gendron, 2001) capture sous une formule discutable (car les monnaies sociales ne sont pas en tant que telles des mouvements sociaux ou l'expression de mouvements sociaux) les finalités critiques de nombre de modèles de monnaies sociales : il s'agit de promouvoir des pratiques d'échange voire de production différentes dans des objectifs de transformation sociale et parfois politiques. Derrière cet objectif, les monnaies sociales souhaitent contribuer à la démocratisation économique par la réappropriation des outils économiques dont les citoyens ne seraient pas maîtres. Les valeurs sont ainsi au fondement de l'innovation sociale qui vise une transformation des pratiques économiques. Une telle logique est en effet au cœur des modèles de monnaies sociales présentés, et de façon très claire au cœur de la monnaie SOL dont l'ingénierie complexe a pour objectif premier de promouvoir un autre rapport aux richesses et donc à la consommation. Le SOL renvoie d'ailleurs fortement à la notion de " consumérisme politique » (Fare, 2009), c'est-à-dire à une consommation engagée reconnaissant le 
pouvoir politique dont disposent les consommateurs (Micheletti, 2004). Le consumérisme politique s'inscrit dans le champ de l'action collective (Stolle et al., 2003), l'acte individuel de consommation étant subordonné à la construction d'une forme d'action collective allant dans le sens d'une re-collectivisation du choix (Dubuisson-Quellier, 2008). Le consumérisme politique des dispositifs de monnaies sociales est cependant surtout tourné vers l'espace des acteurs que construit l'usage de la monnaie (c'est-à-dire vers les acteurs intégrés dans le dispositif), à la différence d'actions de consumérisme politique qui visent à interpeller les entreprises, les pouvoirs publics ou les consommateurs sur divers aspects de l'économie.

Ainsi, les dispositifs de monnaies sociales se positionnent fréquemment en opposition à l'égard du modèle économique dominant. Ce positionnement idéologique se traduit par des représentations collectives communes, "proximité cognitive », fondées sur l'introduction de valeurs éthiques, sociales et/ou environnementales comme fondement de la création du dispositif. Ce positionnement est plus ou moins radical : d'une intégration de valeurs environnementales, sociales et éthiques dans le lien marchand et la production (l'exemple du SOL) à la volonté d'un retrait total à l'égard du marché et des dispositifs marchands (c'est le cas de la plupart des SEL).

\section{L'émergence de règles et de formes novatrices}

L'innovation sociale contient enfin un travail sur les règles et les formes qui est susceptible d'aboutir à la production d'un nouveau cadre normatif (Alter, 2002) fondé sur des valeurs différentes telles que la solidarité, l'égalité, la liberté et la réciprocité. Un tel cadre normatif nouveau est d'autant plus nécessaire lorsque le projet se veut en rupture à l'égard d'un modèle dominant. Il peut s'agir d'établir des formes de gestion novatrice (autonomie de gestion, formes démocratiques de gestion, répartition des pouvoirs et responsabilité) ou d'éloigner les échanges du marché.

Parmi les dispositifs de monnaies sociales, certains instaurent des formes de solidarité - réciprocité dans l'échange monétarisé : dans les monnaies parfois dites de crédit mutuel, de type LETS, SEL et banques de temps, où c'est l'échange qui engendre la monnaie, la reproduction indéfinie des échanges internes repose sur la réciprocité multilatérale des échanges (Servet, 1999). D'autres dispositifs, et parfois les mêmes, introduisent même des dimensions redistributives à partir de prélèvements opérés sur les soldes de chacun ou sur la valeur des billets émis. La prise de distance à l'égard des règles de l'échange marchand que suppose un tel mouvement renvoie à une tentative de redéfinition de l'économie, qui est perçue de manière normative comme devant servir les besoins et les aspirations de la société. 
L'exemple type de cet éloignement des échanges du marché est représenté par le principe d'équivalence en temps mis en place partiellement dans les LETS, plus fortement dans les SEL, et totalement dans les Banques de temps et à l'Accorderie ${ }^{9}$. Ce principe essentiel considère que toute heure est égale à une heure quel que soit le travail effectué et le statut de la personne. Deux idées sont constitutives de ce principe : d'une part l'égalité entre les personnes et d'autre part la reconnaissance de toutes les compétences et savoir-faire, même ceux qui sont habituellement non comptabilisés dans l'économie conventionnelle (par exemple les activités domestiques). Un autre exemple significatif d'établissement de nouvelles normes est celui du $\mathrm{SOL}$. Le système SOL est fondé sur des valeurs sociales et environnementales et cherche à instaurer de nouvelles règles et relations entre offre et demande, entre producteurs et consommateurs ou entre prestataires et usagers. Il en résulte de nouvelles règles et des formes nouvelles de coopération comme la fidélisation des clients (via le crédit porté au compte SOL d'un porteur de carte lorsqu'il passe en caisse d'une structure adhérente) ou l'agrément (l'acceptation de l'adhésion des structures par un comité d'agrément dépend de critères qui ne sont pas strictement définis mais doivent déceler la compatibilité de ses valeurs et de ses perspectives avec celles de la monnaie $\mathrm{SOL}$ ) afin de développer la resocialisation du lien marchand.

En outre, dans chaque type de monnaies sociales se pose la question de l'organisation technique de la comptabilisation des échanges et de leur règlement. À ce titre, on peut noter des formes d'innovation technologique par le développement de logiciels libres (par exemple le logiciel Cyclos développé par l'ONG néerlandaise Aktie Strohalm), la mise en œuvre de plateformes électroniques pour organiser et gérer un dispositif (par exemple le flowplace pour les systèmes d'open money), le recours à des cartes à puces qui suppose un équipement technique des acteurs assez lourd (monnaie SOL en France) ou encore le développement de services de règlement à distance via la téléphonie mobile (Community Exchange System, type de LETS né en Afrique du Sud).

Si l'innovation sociale peut être le résultat d'aspirations à une autre société, la transformation de celle-ci n'est pas nécessairement recherchée. En outre, dans la mesure où l'innovation sociale est un processus se caractérisant par différentes étapes (l'identification des besoins par des acteurs locaux, une mobilisation des acteurs et des ressources, la pérennisation puis la diffusion), seule la dernière étape, celle de la diffusion, peut engendrer une transformation du système. L'innovation sociale doit s'inscrire dans la durée

9. Cahn (2004) conte à quel point la proposition même d'un tel principe d'échange paraissait absurde et dangereuse aux économistes à qui il l'évoquait dans les années 1980. 
(Bouchard, 2006b) et ses effets doivent devenir permanents afin qu'elle devienne un outil de transformation sociale. Des changements macrosociaux attestent d'une transformation sociale; or les innovations sociales se produisent d'abord à micro-échelle, du fait de leur ancrage local et de leur adaptation aux contraintes locales. En outre, elles se déploient plus par réplication (avec plus ou moins de différenciation permettant le développement de nouvelles innovations sociales) que par grossissement. Mais les innovations sociales peuvent être constitutives de la transition entre un modèle de développement et un autre (Lévesque, 2005).

\section{CONCLUSION}

L'émergence et la diffusion de monnaies sociales et complémentaires dans le monde depuis les années 1980 ne se sont pas déroulées selon un modèle unique qui se serait dupliqué à l'infini, mais à partir d'un processus de multiplication et de différenciation. Ce double processus a donné naissance à des modèles variés de monnaies sociales. Ainsi, à partir de l'innovation majeure qu'a représentée la naissance des LETS au début des années 1980, d'autres innovations de plus ou moins grande importance ont donné lieu à une grande variété de dispositifs. Les monnaies sociales mobilisent de manière remarquable différentes formes d'innovations en les combinant : en matière technologique, dans le domaine organisationnel et plus particulièrement celui des modes de gouvernance, dans le rapport aux institutions publiques et dans la mobilisation de règles nouvelles. Mais les monnaies sociales vont au-delà de ces combinaisons : en s'ancrant dans des besoins de proximité dont l'identification est à la fois l'origine et l'objectif de ces dispositifs, elles constituent à part entière des innovations sociales.

L'emballement en matière de débats et de projets de monnaies sociales qui apparaît depuis le milieu des années 2000 laisse augurer l'émergence d'autres modèles, certains franchissant des limites jusqu'ici quasiment intouchées : d'une part, la soumission à des logiques politiques de collectivités publiques, d'autre part, l'intégration dans l'univers des firmes à but lucratif.

Jusqu'ici, cependant, les collectivités locales et les pouvoirs publics sont restés très en retrait de la dynamique des monnaies sociales, se contentant généralement de valider, a posteriori, sur le plan local ou national les innovations émanant de la société civile organisée en associations - bien que les collectivités n'aient pas toujours été favorables à ce type d'innovations, et que les autorités bancaires les aient souvent regardées d'un œil soupçonneux. Un des enjeux de l'institutionnalisation des monnaies sociales, c'est-à-dire « le travail de mise en reconnaissance de l'usage social qui est fait de l'invention ou 
de la découverte » (Fontan, 2007) souligne la nécessité pour les collectivités locales et les pouvoirs publics de soutenir ces dispositifs et de collaborer à leur émergence et développement, sans pour autant casser les dynamiques innovantes qui les ont fait naître.

\section{BIBLIOGRAPHIE}

ALTER, N., 2002, Les logiques de l'innovation : Approche pluridisciplinaire, Paris, La Découverte. BLANC, J., 2002, L'internationale des monnaies sociales, Colloque de l'AISLF « Sociologie économique », 29 mai, Montréal, Canada.

BLANC, J., 2006, Introduction générale. Les monnaies sociales : un outil et ses limites, in Blanc, J. (dir.), Exclusion et liens financiers : Monnaies sociales, rapport 2005-2006, Paris, Economica, 11-23.

BLANC, J., 2011, Classifying "CCs": Community, complementary and local currencies' types and generations, International Journal of Community Currency Research, 15, Special Issue: Complementary currencies: state of the art, 4-10.

BOUCHARD, M., 2006a, De l'expérimentation à l'institutionnalisation positive : l'innovation sociale dans le logement communautaire au Québec, Annals of Public and Cooperative Economics, 77 (2), 139-166.

BOUCHARD, M., 2006b, L'innovation sociale en économie sociale, Montréal, CRISES, Collection Recherche, R-2006-01.

BOUBA-OlGA, O., GROSSETTI, M., 2008, Socio-économie de proximité, Revue d'économie régionale et urbaine (RERU), 3, 1-18.

CAHN, E., 2001, On LETS and Time Dollars, International Journal of Community Currency Research, 5.

CAHN, E., 2004, No More Throw Away People: The Co-Production Imperative, (2000), $2 \mathrm{~d}$ edition, Washington, Essential Books.

CHAMBON, J., DAVID, A., DEVEREY, J., 1982, Les innovations sociales, Paris, PUF, collection Que sais-je ?, 2014.

DEMEULENAERE, S., 2000, Reinventing the market: Alternative currencies and community development in Argentina, International Journal of Community Currency Research, 4.

DOUTHWAITE, R., 1996, Short Circuit. Strengthening Local Economies for Security in an Unstable World, Foxhole, Greenbooks.

DUBUISSON-QUELLIER, S., 2008, De la souveraineté à la gouvernance des consommateurs : l'espace du choix dans la consommation, L'Économie politique, 3 (39), 21-31.

FARE, M., 2007, Le rôle des réseaux dans la performation d'une idée : l'histoire du projet SOL, une monnaie à utilité sociale et écologique, Mémoire de master recherche, spécialité Histoire des théories économiques et managériales, Université Lumière Lyon 2, Lyon.

FARE, M., 2009, Les monnaies complémentaires, des outils au service de la RSE ? $4^{\circ}$ congrès du RIODD : La RSE : une nouvelle régulation du capitalisme, Lille.

FARE, M., 2009-2010, L'Accorderie (Québec) : un dispositif de monnaie sociale singulier ?, Économie et Solidarités, 40 (1-2), 2-16. 
FERREIRA, A., MOERS, P., 2006, Le projet Fomento à Fortaleza, in Blanc, J. (dir.), Exclusion et liens financiers : Monnaies sociales, rapport 2005-2006, Paris, Economica, 355-382.

FONTAN, J.-M., 2007, Innovation et changement social, in Klein, J.-L., Harrisson, D. (dir.), L'innovation sociale : émergence et effets sur la transformation des sociétés, Québec, Presses de l'Université de Québec, 405-412.

GENDRON, C., 2001, Émergence de nouveaux mouvements sociaux économiques, Revue Pour, 172, 175-181.

JACOB, J., BRINKERHOFF, M., JOVIC, E., WHEATLEY, G., 2004, HOUR Town - Paul Glover and the Genesis and Evolution of Ithaca HOURS, International Journal of Community Currency Research, 8, 29-41.

LAACHER, S., 2003, Les SEL. Une utopie anticapitaliste en pratique, Paris, La Dispute.

LAVILLE, J. (dir.), 2007, L'économie solidaire, une perspective internationale, Paris, Hachette.

LAVILLE, J., 2010, Politique de l'association, Paris, Le Seuil.

LÉVESQUE, B., 2005, Innovations et transformations sociales dans le développement économique et le développement social : approches théoriques et méthodologiques, Montréal, CRISES, Collection Etudes théoriques, 507.

LUZZI, M., 2005, Réinventer le marché ? Les clubs de troc face à la crise en Argentine, Paris, L'Harmattan.

MELO, J., 2009, Viva Favela! Quand les démunis prennent leur destin en main, Paris, Michel Lafon.

MICHELETTI, M., 2004, Le consumérisme politique, une nouvelle forme de gouvernance transnationale?, Sciences de la société, 62, 118-142.

NUSSBAUMER, J., MOULAERT, F., 2007, L'innovation sociale au cœur des débats publics et scientifiques, in J Klein, J.-L., Harrisson, D. (dir.), L'innovation sociale : émergence et effets sur la transformation des sociétés, Québec, Presses de l'Université de Québec, 71-88.

PACHE, A.-C., CHALENCON, G., 2007, Changer d'échelle. Vers une typologie des stratégies d'expansion géographique des entreprises sociales, RECMA. Revue internationale de l'économie sociale, 305, 32-48.

RICHEZ-BATTESTI, N., 2008, Innovations sociales et dynamiques territoriales : une approche par la proximité, Marché et Organisations, $\mathrm{N}^{\circ}$ spécial : Développement durable et territoires : économie sociale, environnement et innovations, 7, 61-87.

SERON, S., 1995, Local Exchange Trading Systems, Mémoire de maîtrise LEA, Université de Bourgogne, Dijon.

SERVET, J.-M. (dir.), 1999, Une économie sans argent : les systèmes d'échange local, Paris, Le Seuil.

SEYFANG, G., SMITH, A., 2007, Grassroots innovations for sustainable development: Towards a new research and policy agenda, Environmental Politics, 16 (4), 584-603.

STOLLE, D., HOOGHE, M., MICHELETTI, M., 2003, Political consumerism: A new form of political participation? An exploratory study in Canada, Belgium and Sweden, ECPR Joint Sessions, Edinburgh. 